\title{
Affektive Störungen
}

\section{Depression auch bei schizophrenen Psychosen ausgeprågt}

- Daten aus Psychopathologie, Neurobiologie und Genetik sprechen für eine erhebliche Überlappung von schizophrener Psychose und Depression - eine Herausforderung für die Differenzialdiagnose und eine individuell abgestimmte Therapie.

In vielem glichen sich Patienten in der Prodromalphase von Schizophrenie und Depression, erläuterte Professor Heinz Häfner, ehemaliger Direktor des Zentralinstituts für Seelische Gesundheit, Mannheim, anhand von Ergebnissen der ABC-Schizophrenie-Studie. Nur die Positivsymptome seien einige Monate vor der eigentlichen Erkrankungsepisode bei Schizophrenie wesentlich ausgeprägter als bei Patienten mit Depression. Depressivität sei in beiden Fällen in der Prodromalphase häufig und bei Schizophrenie auch im gesamten Verlauf die häufigste Symptomdimension. „Wir müssen der Behandlung depressiver Symptome mehr Aufmerksamkeit widmen“, forderte Häfner.

In Kliniken erfolge die Therapie häufig mit Blick auf Syndrome wie die Depressivität, aber nicht immer indikationsbezogen. In der Praxis sei das aber so gar nicht möglich, weil die stationär gewählte Medikation für die jeweilige Indikation off-label ist, erklärte Professor Göran Hajak, Bamberg. Umso interessanter seien Therapieoptionen, die ein breites Indikationsspektrum ab- deckten. So sei retardiertes Quetiapin (Seroquel Prolong $^{\oplus}$ ) zur Therapie von Schizophrenie, Manie und Depression bei bipolarer Störung und zur Behandlung depressiver Erkrankungen (Episoden einer Major Depression) als Zusatztherapie bei Patienten zugelassen, die unzureichend auf die Monotherapie mit einem Antidepressivum angesprochen hätten. Die Leitlinie der World Federation of Societies of Biological Psychiatry zur Therapie bipolarer Depression empfehle sogar Quetiapin, nicht Antidepressiva, als Monotherapie mit dem höchsten Evidenzniveau (A1) [1]. Ein Problem sind derzeit laut Hajak die unterschiedlichen zugelassenen Dosierungen von Quetiapin in den verschiedenen Indikationen. In den meisten Fällen helfe aber die Standarddosis [2] . Das Motto „Ein Medikament für alles“ könne aber nicht gelten: Wesentlich sei die klare Definition des Behandlungsziels mit dem individuellen $\mathrm{Pa}$ tienten und die daran orientierte Medikation gemäß Zulassung.

Friederike Klein

1. Grunze H et al. World J Biol Psychiatry (2002) 3: 115-124 2. Sparshatt $S$ et al. CNS Drugs (2008) 22: 49-68

Satellitensymposium „Personalisierte Therapie der Schizophrenie und affektiver Störungen - was sind die Erfolgsfaktoren", DGPPN-Kongresses 2011, Berlin, 24.11.2011 Veranstalter: AstraZeneca GmbH

\section{Alzheimer-Demenz}

\section{Therapeutische Optionen kombinieren}

- Heute leben in Deutschland etwa 1,3 Millionen Menschen mit einer Alzheimer-Demenz, bis zum Jahr 2050 wird sich die Zahl der Krankheitsfälle verdoppelt haben [1]. Welche Therapiekombinationen sind optimal?

Für leichte bis mittelschwere Stadien der AlzheimerDemenz sind Acetylcholinesterase-Hemmer (z.B.: Donepezil $^{\circledR}$ ) geeignet, für moderate bis schwere Stadien Memantine (z. B: Ebixa ${ }^{\oplus}$ ). Nach Datenlage und Praxiserfahrung gilt laut Professor Rainer Hellweg, Berlin: „Ein früher Therapiebeginn ist besser als ein späterer und ein späterer Therapiebeginn ist besser als keine Therapie“. Die Wirksamkeit von Memantine belegen erneut die aktuell veröffentlichten Responderanalysen [2]. Für die Kombinationstherapie aus Memantine und Donepezil werden synergistische Effekte postuliert; diese Verstärkung zeigte unter anderem eine 24-Wochen-Studie [3]. Es könne sich lohnen, Patienten mit moderater bis schwerer Alzheimer-Demenz kombiniert zu behandeln, so Hellweg.

„Individuelle nicht-medikamentöse Maßnahmen sind als Zusatz zur Arzneimitteltherapie ein wichtiger Baustein der Behandlung“, betonte Dr. Brigitte GrassKapanke, Krefeld. Als Beispiele nannte Grass-Kapanke
Bewegungstherapien, kognitives Training, kreatives Arbeiten, Selbsterhaltungstraining, Schulung der Angehörigen und Umfeldgestaltung. Die verschiedenen Optionen sollten individuell für jeden Patienten entsprechend seiner Persönlichkeit und dem Schweregrad seiner Erkrankung ausgewählt werden. Eine Überforderung - etwa im kognitiven Training - könnte andernfalls zu Frustration, Scham, Rückzug oder auch Aggressivität führen. Individuell passende Angebote hingegen könnten zusätzlich zu dem Übungseffekt auch das Selbstwertgefühl erhöhen. Und gerade der Erhalt des Selbstwertgefühls und die Vermeidung der Scham seien für Demenzpatienten wesentlich, betonte GrassKapanke.

Dr. Friederike Holthausen

1. Berlin-Institut für Bevölkerung und Entwicklung, 2011: Demenz-Report

2. Hellweg R et al. 2011, Int J Geriatr Psychiatry, doi:

10.1002/gps.2766

3. Tariot PN et al. JAMA 2004; 291: 317-24

Lundbeck-Expertengespräch DGPPN 2011 „AlzheimerDemenz: Welche Therapien sind optimal für den Patienten?", DGPPN-Kongresses 2011, Berlin, 25.11.2011 Veranstalter: Lundbeck GmbH, Hamburg
Berichte von Symposien der Pharmaindustrie 